CAHIERS DE

NARRATOLOGIE

\section{Cahiers de Narratologie}

Analyse et théorie narratives

$19 \mid 2010$

Images composites, arts pluriels

\title{
La forme-balade du travelling et autres modes d'incursions mouvementées dans le film Prospero's Books
}

\section{Patrick Louguet}

\section{(2) OpenEdition}

Journals

Édition électronique

URL : http://journals.openedition.org/narratologie/6175

DOI : 10.4000/narratologie.6175

ISSN : 1765-307X

Éditeur

LIRCES

Référence électronique

Patrick Louguet, « La forme-balade du travelling et autres modes d'incursions mouvementées dans le film Prospero's Books », Cahiers de Narratologie [En ligne], 19 | 2010, mis en ligne le 22 décembre 2010, consulté le 02 mai 2019. URL : http://journals.openedition.org/narratologie/6175 ; DOI : 10.4000/ narratologie. 6175

Ce document a été généré automatiquement le 2 mai 2019.

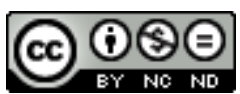

Cahiers de Narratologie - Analyse et théorie narratives est mis à disposition selon les termes de la licence Creative Commons Attribution - Pas d'Utilisation Commerciale - Pas de Modification 4.0 International. 


\title{
La forme-balade du travelling et autres modes d'incursions mouvementées dans le film Prospero's Books
}

\author{
Patrick Louguet
}

Auto-génération du cinéma:

il faut que les films engendrent d'autres films

Dziga Vertov

\section{Un paradoxe}

\section{La proclamation de la mort du cinéma, mais aussi son affirmation comme art de l'artifice}

\section{Les parcours urbains}

Pour qui n'a pas eu la chance d'assister au colloque niçois organisé en février 2009 en présence du cinéaste lui-même, il est quand même possible de rencontrer Peter Greenaway et de recueillir ses propos dans le précieux bonus du DVD consacré au film Le cuisinier, le voleur, sa femme et son amant édité tout récemment, en décembre $2008^{1}$. On dispose également du film documentaire artistique de 2004 intitulé Peter Greenaway, Portrait ${ }^{2}$ et désigné comme "film entretien » par Philippe Pilard et son collaborateur Jean-Jacques Bernard, dans l'un des cartons du générique. Dans cette suite est inséré un gros plan du créateur anglais qui énonce d'entrée de jeu, avec la tonique mauvaise foi qui le caractérise : «Le cinéma est mort ». Cette tonique mauvaise foi est renforcée par la brièveté du plan mais sera nuancée lorsque l'artiste mettra en exergue la capacité de l'art du cinéma à faire advenir des mondes artificiels propres à nourrir l'imaginaire des spectateurs, à la suite immédiate d'un propos de son infographe Eve Ramboz. Celle-ci souligne que le plan mouvementé, de courte durée, du ricochet de la goutte d'eau, est une image composite formée de l'association de cinquante sources iconiques. Il convient 
d'ajouter au passage, pour attester de la vigueur polémique de la problématique, que Pierre Huygue - estimant tout à fait secondaire la question des supports et qui tient à ce que ses œuvres soient projetées sur grand écran en diverses expositions d'art contemporain - a résolu la question une fois pour toutes en déclarant «Je ne m'intéresse pas à la question du médium. Je fais des films, je ne fais pas de vidéos ${ }^{3}$ ”

2 En outre, dans le documentaire de Pilard - avant qu'un carton de générique de fin ne rende hommage au grand chef opérateur Sacha Vierny ${ }^{4}$, que Greenaway admirait tant au point de se le partager pendant un temps avec Alain Resnais - il affirme que si le cinéma est un « dinosaure » dont la tête peut sembler figer, il faut se méfier de « la vivacité de sa longue queue ». Le «film entretien » de Pilard est tourné à Gand en 2006 et les différents propos sont entrecoupés de la captation d'un tournage de Greenaway d'un opus intitulé Les valises de Tulse Luper ${ }^{5}$, de telle sorte que le documentaire de Pilard se présente aussi comme son making of. Les valises ont d'abord été présentées dans une installation gigantesque de «Lille 2004, capitale européenne de la culture » ${ }^{6}$ À Gand, la caméra numérique à très haute définition de Greenaway trouve ses marques, non pas au musée d'art contemporain belge, le SMAK, maisdans une galerie privée très spacieuse, la galerie « Fortlaan $17 »^{7}$. Du film à l'installation intra ou extra muros et de l'installation au film, il y a un va-et-vient incessant dans l'œuvre d'ensemble de Peter Greenaway et l'on se souvient aussi des installations The Stairs, qui parsemaient Genève seulement trois ans après la sortie de Prospero's Books. L'année qui a suivi la sortie de Prospero..., on peut aussi faire état de l'installation viennoise «Cent objets pour représenter le monde». Ces installations tiendraient du jeu de piste si elles n'entraient dans des combinatoires multiples de libres parcours. La marche et la flânerie sont consubstantielles au $7^{\mathrm{e}}$ art, ainsi que Suzanne Liandrat-Guigues en administre les preuves sur de nombreux films dans son dernier ouvrage, Modernes flâneries du cinéma ${ }^{8}$ etl'arpenteur erre dans la ville sans autre but que le plaisir éprouvé à la déambulation. Dans le cas des parcours urbains de Greenaway, l'artiste agit comme un metteur en scène à une vaste échelle et le marcheur n'est pas un flâneur. Détenteur ou opérateur de cause finale, le concepteur de multiples dispositifs urbains de sélection de points de vue est en effet celui qui impose aux promeneurs - devenus spectateurs - des coups d'arrêts qui sont comme autant de coups de force imposés au regard, contraint à un cadrage parfois étriqué.Ce cadrage, que le spectateur urbain mérite, lorsqu'il monte par exemple sur la petite estrade d'une installation, n'offre jamais l'équivalent d'un très gros-plan, quoiqu'on en ait dit. En effet l'immersion corporelle du regardeur, dans une situation urbaine, ne parvient pas à se faire oublier aussi aisément que dans la salle de cinéma, lorsqu'il fait face au grand écran et qu'il éprouve des sensations et émotions sensibles à partir de la position assise. Le spectateur-promeneur circule dans des installations disséminées dans la ville de telle sorte que la ville toute entière révèle sa nature profonde d'installation interactive, alors que lors du retour sur écran les événements ne dépendent plus que du seul bon vouloir du réalisateur, pour le plus grand plaisir du spectateur de salle. C'est vrai que, face aux activités organisationnelles et créatrices multiformes de Greenaway, il est important de souligner les va-et-vient plutôt que de se poser la question d'un possible renoncement à l'art du cinéma, comme s'il ne s'agissait pas de nécessité intérieure. Sans vergogne, en 1987, un journaliste de Libération ose lui poser la question impertinente, et immature dans la bouche d'un adulte, «Pourquoi filmez-vous ?» Greenaway a la générosité de ne pas en relever l'incongruité et de répondre :

Peut-être que je fais des films pour pouvoir écrire et peindre en même temps... et faire des listes... et inventer des catalogues... et réfléchir aux possibilités et 
rapporter des paradoxes, développer des ironies, citer des énigmes, inventer des

citations, raconter des histoires (...)

3 À considérer ce qui suit, on va s'apercevoir qu'entre 1987 et 1995 l'art du cinéma de Greenaway s'émancipe de plus en plus du narratif.

\section{Support narratif et laboratoires de transsubstantiation}

Dans l'art du cinéma de Peter Greenaway, la narration est devenue inessentielle en ce qu'elle n'est plus qu'un simple support, un prétexte squelettique destiné à jouer le rôle de "lien-liant", un matériau destiné à exalter l'ordre du figural comme tel. Si Prospero's Books respecte apparemment le tracé d'une ligne narrative, entre la première arrivée dans l'île et la venue tardive du frère usurpateur accompagné du roi de Naples et de son fils (lequel, évidemment, s'éprendra de Miranda, la fille du duc), c'est pour mieux pouvoir emprunter les chemins de traverse et forger des matrices d'édification de formes qui répondent au génie du cinéaste ${ }^{10}$. Car si l'histoire joue un rôle de liant, si squelettique soit-elle, elle n'est évidemment pas le seul agent d'édification sous-jacent, et bien plus motivants sont les squelettes d'animaux et les charpentes plus ou moins gangrénées des constructions artificielles.

Pour être juste, il faut dire que la promesse de récit - entre l'ouverture d'un prologue et le dénouement d'un épilogue - est tenue. Toutefois, ce qui déconcerte les spectateurs attachés au confort d'une narration classique (bien loin de la méthode du cut up préconisée au milieu du XXe siècle par le peintre et poète Brion Gysin et pour laquelle l'écrivain américain William Burroughs se passionna) c'est probablement les chemins de traverse et les proliférations composites spatio-temporelles auxquelles, précisément, ils donnent accès. C'est qu'alors la toile cinématographique est devenue le lieu de collages permanents, témoignant de la persistance et de la vigueur des principes avant-gardistes, dadaïstes et cubistes (avant de devenir surréalistes), affirmés pour le $7^{\mathrm{e}}$ art.

Que le duc de Milan erre sur mer, avec sa fille, dans une barque au bois pourri, en une circulation inaugurale, place le film d'entrée de jeu, dès l'accostage, sous l'égide de trois motifs - disons les humanistes - hérités de la Renaissance, avec cependant des traces de conceptions moyenâgeuses: la vanité, la sensualité livresque exaltée dans les enluminures, et la magie. Que des significations surnaturelles soient à l'œuvre dans ce film qui ne contredisent en rien l'humanisme de la Renaissance n'est pas pour étonner quand on sait que, pour Michel Foucault, l'épistémè de la Renaissance - qui précède celle, " hyper-rationaliste », de l'âge classique - est celle qui se caractérise en tout domaine par «le jeudes similitudes et des ressemblances». Le jeu des correspondances "sympathiques », micro et macrocosmiques, peut même s'établir entre ordres naturels et ordres surnaturels, très significativement pour ce qui concerne l'art et la religion ${ }^{11}$. Le fond mythologique de Greenaway, comme celui de Shakespeare, faisant se côtoyer mortels et immortels, est franchement et joyeusement polythéiste qui puise aux sources antiques de Babylone et d'Athènes pour ce qu'il en est de diverses métamorphoses. Cela ne l'empêche d'ailleurs pas d'emprunter des motifs à certains récits venus du moyen-âge. Les fééries dominent donc la tempête ${ }^{12}$ et permettent d'affirmer la connivence entre la poétique théâtrale de Shakespeare et celle, cinématographique, de Greenaway, dans une alliance de bon aloi qui rend vaine toute affirmation d'anachronisme. Dans l'énergie exubérante de l'art théâtral de Shakespeare, dans ses foisonnements protéiformes qui ont aussi conduit ses figures et motifs vers le chant et la danse de scènes d'opéra, il y a les 
racines tout aussi impures que canailles de l'art du cinéma ${ }^{13}$. On le sait bien désormais, avec les travaux d'un Jean-Louis Leutrat ou d'un Stanley Cavell - revenant, entre autres, sur la modernité baudelairienne - le désir de cinéma s'enracine dans de multiples sources et dispositifs depuis l'antiquité et n'a pas attendu les conditions techniques de sa réalisation pour se manifester ${ }^{14}$.

7 Évidemment, dans cette rencontre entre Shakespeare et Greenaway, avec les opérations de tours de magies réalisées à vue, Méliès, mais aussi Marey et Muybridge, apparaissent comme de véritables intercesseurs, au sens deleuzien du terme ${ }^{15}$. C'est donc du côté de l'art de l'image-mouvement et de l'image-temps qu'il faut chercher la spécificité des apports créatifs de Greenaway, et non du côté d'on ne sait quelle séduction narrative, laquelle est désavouée par l'artiste de la maturité. C'est que les charmes déployés dans ses œuvres nous conduisent loin des enfantillages pour considérer les subtils entrelacements, tout aussi matiéristes qu'existentiels entre la vie, l'amour, la sexualité et la mort ${ }^{16}$. Ces entrelacements sont tellement forts, tellement denses, qu'ils ont déconcerté une certaine critique grand public en 1991. Celle-ci se faisait la porte parole complaisante des déçus du narratif et utilisa les vocables - pour elle méprisants - d'art "futile", "trop baroque" ou encore "formaliste". Évidemment, accueillir ses films présuppose qu'on le fasse sur très grand écran et qu'on garde, face à eux, les yeux grands ouverts ${ }^{17}$. Dès lors, pour caractériser l'appel à la disponibilité du regardeur éprouvant de toutes nouvelles perceptions, on pourra reconduire le mot de Bill Viola quand, dans les années 70, au centre culturel américain de Paris, on lui posa la question : "Où est la narration dans cette vidéo?». Viola répondit: «La narration, c'est l'œuvre ${ }^{18}$ ! »Si Greenaway met en scène les livres de Prospero, ce n'est pas pour que nous les lisions, c'est pour utiliser leur matérialité comme source d'une image composite, c'est pour explorer les valeurs figurales de la lettre prise dans des processus naturellement mouvementés d'apparition, d'effacement ou de disparition, à l'instar de ceux qui affectent les éléments naturels. Affirmée en une première couche, la lettre ornementée ou calligraphiée permet à Greenaway de réaliser cinématographiquement un véritable "devenir-palimpseste", à rapprocher, mutatis mutandis, de l'érosion qui s'empare du nom de Madame Muir gravé dans le bois, le panneau de la plage étant véritablement taraudé par les vagues, et cela durant de longues années, dans le film éponyme de Manckiewicz ${ }^{19}$.

Les livres-accessoires de Prospero, tels qu'appropriés par le réalisateur anglais, sont alors à comprendre comme de véritables boîtes à malice et non comme des ensembles de feuilles de papier qui affirmeraient le primat de la sémantique sur la sémiotique. Car, ce qui est déterminant, c'est bien la conquête et l'invention de nouvelles configurations sensibles, prises toutes entières dans le jeu des signes visuels et sonores. Du côté d'une sémiotique de l'accessoire - des ses valeurs métaphoriques si l'on veut, à condition de parler comme Jacques Gerstenkorn de «métaphore visuelle ${ }^{20}$ »- la séduction cinématographique du grand livre, outre son «devenir-palimpseste » et sa combustion lente ou rapide, s'exerce évidemment dans les circulations de ses pages. Le grand livre s'impose alors comme matrice ou schème mouvementé de temporalitéé ${ }^{21}$, dans un «devenir-clepsydre» en quelque sorte (passer d'un livre à l'autre équivaut bien à la consultation d'un seul grand livre virtuel que le film actualise en ses différentes phases épisodiques) : Greenaway revivifie, ou décline à sa façon, l'effeuillage des éphémérides, capté le plus souvent en très gros plan, dès l'époque du cinéma muet.

9 Pour rebondir sur la question de la narration subordonnée, secondaire voire inessentielle, citons des propos de Greenaway lui-même, dans l'interviewaccordée à artpress en 1995. 
Je pense que Griffith a sans doute fait beaucoup de tort au cinéma. En un sens le cinéma s'est complètement fourvoyé en prenant pour support le récit. Rien n'oblige le cinéma à être narratif alors que tout l'oblige à être séquentiel ${ }^{22}$. Les catalogues sont un des traits distinctifs des films que j'ai faits, et a fortiori du film que je suis en train de faire (The Stairs) : dresser des catalogues, réduire le récit à sa plus simple expression, tenter d'analyser ce que la peinture a apporté. Les chefs d'œuvre de la peinture ne sont pas narratifs, ils ne font qu'émettre un point de vue sur certaines façons de concevoir le monde, le fond ou la forme ${ }^{23}$.

On adhère évidemment à ce propos de Greenaway quand il énonce que «Rien n'oblige le cinéma à être narratif». En revanche, comme souvent avec ses jugements à l'emportepièce, on ne saurait souscrire entièrement à ce qu'il disait de Griffith à l'époque car insister sur les grandes lignes de récits de Naissance d'une Nation ou les récits alternés d' Intolérance - quittés puis repris - ne doit pas conduire à nier injustement de très fortes inventions artistiques dans l'œuvre de Griffith ${ }^{24}$. En tout cas cette interview a le mérite de bien établir que la quête figurale du réalisateur anglais - ses expérimentations qui mobilisent tout autant des solutions de tournage que de postproduction, associant sur banc de montage et à la truca des moyens d'animation image par image, des effets spéciaux traditionnels et des procédés numériques dont celui du balayage au scanner de ses œuvres graphiques - a toujours pour horizon la picturalité et l'affirmation d'un certain pointde vue. Quant au "devenir-palimpseste", il s'origine dans l'art pictural privilégié par Greenaway :

Réduire le récit à sa plus simple expression, tenter d'analyser ce que la peinture a apporté. Les chefs d'œuvre de la peinture ne sont pas narratifs, ils ne font qu'émettre un point de vue sur certaines façons de concevoir le monde, le fond ou la forme ${ }^{25}$.

11 Expérimentation: le mot n'est pas trop fort pour parler des laboratoires de transsubstantiation érigés par le réalisateur anglais. Ces creusets d'édification de formes tiennent autant du laboratoire de l'alchimiste que de ceux du physiologiste ou du physicien. Ainsi, parmi toutes les ressources de l'art de Greenaway, il y a l'exaltation des combustions lentes ou soudaines, dans des événements qui affirment alors leur filiation au petit traîneau du Kane d'Orson Welles - petit traîneau qui dans les flammes est, comme on sait, lieu de révélation au moment même où il disparait - comme d'ailleurs à l'oreiller brodé au nom de Rebecca dans le film éponyme d'Alfred Hitchoock ${ }^{26}$. Il faut dire que les flammes lumineuses, et autres gerbes étincelantes, affirment d'emblée leur connivence mouvementée avec ce qui rend possible la projection cinématographique. Il faut souligner, une fois de plus, la forte affinité de ces processus filmés avec les accidents de combustion qui, jadis, se produisaient souvent en cabine avec leurs effets en salle, du déraillement de certains projecteurs. Alors, de façon tout aussi intempestive que brutale, la pellicule était dévorée à partir du centre de l'image, sous les yeux tout aussi ébahis que fascinés des spectateurs qui assistaient à de tels accidents. Pour reprendre, avec Julie Savelli, le titre de la rubrique de Jean-Claude Biette dans la revue Trafic il y a bien chez Greenaway cette capacité propre à tout grand réalisateur d'exalter en chacun de ses films un cinéma "à pied d'œuvre ${ }^{27}$ ", capable de rassembler toutes ses ressources, de la captation à la projection, en passant par toutes les étapes "artistico-techniques" de la post-production. À tout moment, l'artiste - grâce à ses combinaisons les plus originales, par et dans ses formes les plus achevées, et en dépit de la narration - permet au spectateur d'accéder à cette "obscure conscience filmante " que son œuvre a d'ellemême, comme le dit aussi si justement Julie Savelli ${ }^{28}$ Ici, avec la prise en compte des multiples «gestations » préparatoires de l'infographe Eve Ramboz, on peut ajouter que 
l'image-mouvement de nature composite affirme sur l'écran, tantôt ses traces d'édification ou son hétérogène constitutif, tantôt, au contraire, se donne comme un bloc « homogénéisé » avec le parti-pris d'effacer toute jointure ou toute suture des éléments disparates, au bénéfice de l'invention de chimères cinématographiques. Restent diverses possibilités offertes au réalisateur de "faire sale" en donnant éventuellement à voir quelques pixels ou en "floutant" quelques zones de la surface, ou encore en portant des coups de brosse aux contours indécidables, scannés comme tels avec la trame ou le grain de leur support. Il faut aller y voir au cas par cas, comparer Prospero... aux valises de Tulse Lupper, mais il semble bien que dans l'opus de 1991 c'est le parti pris d'homogénéisation des formes mouvementées qui s'impose. Ce parti-pris est affirmé dès le travelling du générique, lequel revêt une fonction de modélisation propre au $7^{\mathrm{e}}$ art.

\section{Le point de vue constamment déplacé}

\section{Variabilité de l'audience camera}

Et pourquoi s'étonner que le film soit, de part en part, habité par l'« obscure conscience » filmante d'un work in progress quand la question essentielle, est celle du point de vue ${ }^{29}$. Si point de vue de cinéma il y a, celui-ci ne pourra être que mobile, en déplacement sur des objets et événements eux-mêmes en mouvement tout à fait en accord avec la thèse deleuzienne selon laquelle le cinéma c'est une coupe de mouvements d'autant plus fluide qu'elle est elle-même mobile. Du coup l'atomisation instantanéiste de la photographie ne saurait lui fournir son parengon artistique. L'art du cinéma, dès son origine, est en affinité plutôt avec la sculpture qui, pour Suzanne Liandrat - revenant sur la façon dont Deleuze affirme le bergsonisme - est bien cette série continue de coupes mobiles de mouvements ${ }^{30}$ . Dans cet esprit, pour le livre 18 de Prospero où est évoquée Sémiramis, la reine guerrière de Babylone, Greenaway obtient d'Eve Ramboz, qu'elle mette en mouvement des lutteuses ${ }^{31}$ dans une certaine parenté avec les planches chronophotographiques de Muybridge éditées en de nombreux albums à l'allure de livres d'art. C'est bien alors de préhistoire du cinéma qu'il s'agit et la conscience est ici carrément explicite. Car si la décomposition photographique instantanéiste du mouvement s'approche du cinéma dans sa fixation de très petits intervalles temporels, elle ne saurait en tenir lieu. Philippe Pilard, dans son documentaire, ne s'y est pas trompé qui, dans l'esprit de Greenaway, place immédiatement à la suite de cette lutte extraite du film un modèle féminin, nu de Marey en train de marcher de façon continue, et non une planche photographique des phases décomposées de cette même marche. La convocation qui s'affirme comme un hommage est en même temps arraisonnement profilmique qui suppose continuité de défilement du ruban pelliculaire lors de sa projection sur écran. Cela peut paraître trivial de le rappeler mais ce que Pilard exalte alors, c'est l'animation cinématographique image par image, un des principes fondateurs du cinéma, réalisé dès le début du XXème siècle, s'ajoutant à celui des "coupers-collers" de Mélies qu'on ne peut dissocier de ses "trucs à arrêt ${ }^{32}$ ". L'intérêt de l'image composite de Greenawy-Ramboz c'est d'animer des dessins - ou des photogrammes rendus au dessin par décalque des contours et affectés de divers traitements graphiques qui leur redonnent de l'épaisseur - et de rendre le corps à corps des lutteuses d'autant plus fluide qu'il semble naître de la tension établie entre coups de crayons ou coups de brosses et support lui-même. L'émotion perceptive est alors tout autre que celle de la simple animation des vues chronophotographiques d'où les lutteuses 
semblent provenir. En cette singularité, chaque phase de lutte (corps à corps - empoignades - chutes) s'inscrit dans les traces de la précédente, un peu selon le principe adopté par McLaren en 1967quand il réalise Pas de deux. Dans ce court métrage du réalisateur canadienles corps des danseurs sont affectés d'un véritable « deveniroiseau » dès lors qu'ils se trouvent métamorphosés par les traces-silhouettes, réitérées et maintenues visibles derrière eux, au fur et à mesure de leurs déplacements, par une sorte d'effet stroboscopique.

Les livres de Prospero appartiennent tous à la surface plane surcadrée qui est aussi creuset de métamorphoses, lieu de questionnement de la matière organique, en accord avec une tradition qui va de Rembrandt à Francis Bacon ${ }^{33}$. Mais avant de considérer une nouvelle fois cette "surface-creuset", il convient d'accueillir la deuxième partie du générique d'une durée de quatre minutes car - véritable préalable mouvementé - elle s'impose comme l'une de ses conditions de possibilité. Il s'agit là d'un très long plan séquence où les lettres du générique se succèdent en paquets et sont installées comme grilles translucides d'exacerbation de la visibilité, occupant le plus souvent le cadre qui sera, grosso modo, celui des livres ouverts. Toutefois ces grilles sont virtuelles et se donnent à voir à la manière de stores vénitiens entr'ouverts.

Dominique Païni voit dans le film Lost Highway de David Lynch la réalisation du principe d'une visite en différents lieux d'un même musée ${ }^{34}$. On peut dire que ce principe de la visite par un spectateur qui déambule, en dépit de sa position assise, est à l'œuvre dans le générique de Prospero... La différence essentielle, toutefois, est que l'on visite des espaces contigus et continus, certes comme en certains musées, mais que l'on entre ainsi en différents ateliers de sculpteurs ou de peintres pour en surprendre les modèles vivants ; que l'on visite un lieu d'expérimentations chorégraphique soutenues par la musique de Mickael Nyman; qu'on accède à des espaces théâtraux d'évolution circulaires de comédiens dont certaines figures de groupe ne sont pas sans rappeler les performances du Living Theater, ou qu'on assiste au saut à la corde de deux femmes, en écho avec celui de la petite fille évoquant une ménine de Velasquez dans ce film antérieur qu'est Drowning by Numbers (1988). Cependant, pour Mickael Nyman, le principe natif est d'abord pictural et la musique est là pour parachever l'art cinématographique de Greenaway :

La référence picturale : l'interpénétration de la peinture et du cinéma s'effectue chez Greenaway, non seulement au cœur de l'image et de sa matérialité, mais aussi dans le processus qui lui donne naissance. Travail du peintre et travail du cinéaste se rejoignent dès leur abord technique ${ }^{35}$.

On peut soutenir aussi, complémentairement, que tous les composants hybrides profilmiques s'enracinent dans le musée imaginaire du créateur anglais, éminemment composite. On peut le soutenir, en effet, à condition de dire que ce musée - de l'ordre d'une cosa mentale - est pétri de souvenirs d'images fixes (au premier chef, celles créées par lui) et $\mathrm{du}$ désir de les mettre en mouvement, de les dynamiser cinématographiquement. Ce musée est donc de part en part mouvementé, à l'instar de celui de Lynch, comme de tout réalisateur d'ailleurs. Les figures, motifs et stéréotypes culturels sont très nombreux auxquels le spectateur a accès lors de cette « forme-balade ${ }^{36}$ » du travelling d'une durée de quatre minutes. Comme Suzanne Liandrat le dit de la flânerie, dans l'ouvrage déjà cité, il nous semble que ce travelling continu fonctionne comme « un opérateur de métamorphoses ${ }^{37}$ ». Évidemment, et l'auteure y insiste, il est de l'essence de la flânerie d'être sans but précis ou bien assigné. D'autre part Prospero est toujours très entouré alors qu'on imagine plus volontiers la flânerie comme étant 
conduite solitaire. Mais est-on bien sûr que, déambulant tout au long du film, flanqué de ses livres, Prospero ne s'égare pas, prenant des chemins de traverse, à défaut de ne pas savoir où il va? Les architectures grandioses - celles des salles du palais qu'il arpente devraient conforter l'hypothèse dès lors qu'elles tendent à abolir la frontière entre l'extérieur et l'intérieur. La présence de colonnes multiples dans le décor, comme autant de jalons d'un parcours, nous reconduit tout naturellement aux conceptions de Hegel. Dans son Esthétique, comme on sait, le philosophe allemand met en évidence la parenté entre colonnes et grands arbres, celle à l'œuvre en divers édifices, des temples grecs jusqu'aux voûtes des cathédrales, à l'image des branches aériennes entrelacées dans les vastes forêts.

Là où la critique grand public n'a vu que juxtaposition érudite, symptôme probablement de son refus de se laisser "embarquer", Greenaway opère - grâce à cette forme mouvementée englobante d'une forme-balade qui grossit au fur et à mesure qu'elle avance - une unification de figures à fonction d'universalisation.C'est ainsi que le personnage nu, suspendu la tête en bas, et qui évoque le supplice subi par Machiavel, cède bientôt la place à une scène qui montre Léda et le cygne captés comme des modèles dans l'atelier du sculpteur: Léda nue, accroupie, caressant le cou de l'oiseau. Plus loin on assiste à la déambulation, en une série d'allers et retours combinés au travelling, jusqu'à sa sortie bord gauche, d'un modèle accoutré selon l'un de ceux de Félicien Rops ${ }^{38}$. Il faudra, certes, attendre une autre séquence, centrée sur le rapport électif qui lie à ce moment Miranda à son père pour voir, en arrière plan, le personnage d'Actéon déambuler et s'arrêter sur une marche d'escalier, bord droit, avant d'être entouré par d'autres modèles. La composition chimérique, très présente dans la sculpture, répond depuis l'antiquité à un art composite bien affirmé dans la réalisation de l'homme à tête de cerf. On sait combien elle trouve aussi de puissantes expressions dans les collages surréalistes, par exemple ceux de Max Ernst, mais aussi dans divers agencements fantastiques du $7^{\mathrm{e}}$ art. Il suffit de citer le film Judex de Georges Franju (1964) dont les figures du bal masqué - homme à têtes d'oiseaux - entrent bien en écho avec certaines gravures à l'eau forte du plasticien.

17 Cette déambulation métamorphosante c'est alors, on l'aura compris, une pérégrination au carrefour des arts régie par la seule loi exubérante d'une rencontre entre événements perçus, images-souvenirs et projections mentales. Elle relève aussi de l'ekphrasis, ce protocole de la rhétorique antique par lequel les arts rivalisent, se mesurent les uns aux autres, comme pour mieux éprouver les moyens d'expression qui leurs sont propres ${ }^{39}$. À cet égard, Il y a une parenté évidente entre ce film de Greenaway de 1991 et le film Passion (1982) de Jean-Luc Godard lorsqu'il met à l'épreuve la peinture de genre dans l'édification cinématographique de véritables tableaux vivants.

D'aucuns parleraient peut-être de forme brève de cette deuxième moitié du générique de Prospero... en la rapportant à la durée globale du film. En fait, régie par une durée qui s'entretient à la vitesse constante du travelling - cette "forme-balade" qui réside d'abord dans la déambulation majestueuse et régulière du duc - est d'autant plus longue qu'elle est creuset d'émergence d'autres formes sans qu'entre toutes il y ait rupture. C'est ainsi que, dans la suite du film, Greenaway édifie aussi des travellings continus selon l'axe de la profondeur de champ et que ceux-ci sont régis par la même vitesse, accordée à celle de la musique de Mickaël Nyman: la régularité s'impose, nonobstant les changements d'orientation d'une audience camera en balade.L'art du cinéma étant de combiner différents mouvements dans des blocs de mouvement-durée, on peut dire que le plan- 
séquence de déambulation, près de la fin du film, d'une double cohorte de gens de cours bientôt suivis de modèles nus, a pris le relais des travellings établis en profondeur de champ : les hommes et les femmes partent du background et quittent l'écran à l'avant-plan d'un cadre fixe à ce moment (en passant très près de la caméra, en deux files réparties de part et d'autre). S'agissant de parcours de relative longue durée, on dira que cette prise de relais est aussi un équivalent mouvementé, par quoi s'affirme au cinéma la solidarité relationnelle étroite qui lie toujours personnage et caméra.

Quant au travelling "longitudinal" de quatre minutes, on imagine alors les prouesses techniques se jouant d'accessoires - d'abord profilmiques avant de devenir des obstacles au déplacement de la caméra sur rails - lors de la prise de vue effectuée par le chef opérateur Sacha Vierny. Long travelling: dans les souvenirs artistiques du talentueux chef opérateur, il y a sa collaboration au film d'Alain Resnais de 1959, Hiroshima mon amour, en particulier s'agissant de l'édification d'une ville composite associant des travellings effectués à Nevers et à Hiroshima. Il serait très étonnant que ce film ne fasse pas partie de la culture cinématographique de Greenaway lorsqu'il recrute Vierny en $1987^{40}$.

Ajoutons que ces prouesses à l'œuvre dans le générique de Prospero... sont analogues aux tours de force réalisés par les déplacements, au milieudes nombreux couples de danseurs, de la Louma d'Hitchcock lors du tournage de la séquence finale, au grand hôtel, du film Jeune et Innocent (Young and Innocent - 1937). On peut le dire mutatis mutandis car, dans ce dernier cas, le travelling n'est pas latéral mais creuse l'espace selon l'axe de la profondeur de champ.

21 S'agissant de cette "forme-balade", la filiation à l'œuvre de Godard s'impose une fois de plus quand on sait que lors de la réalisation de Week-end en 1967, le chef de file de la nouvelle vague proclama qu'il allait réaliser «Le travelling continu le plus long de l'histoire du cinéma ", un travelling de 300 mètres. Mais nous dit Alain Bergala, dans son essai Godard au travail ${ }^{41}$, " ce travelling prétendument historique de Godard est coupé en deux par des cartons. Cela annule ainsi l'aspect "morceau de bravoure" quelque pu "m'astu-vu" du plan ${ }^{42} »$. Du coup les onze minutes subissent une coupe franche propre à les diviser lors du travelling effectué le long du bouchon interminable des automobiles, ponctué de scènes d'accidents. Avec l'insertion des deux cartons, Godard obtient alors un premier segment de deux minutes quarante secondes et un second approchant les cinq minutes, comme pour faire oublier que, d'un travelling à l'autre, l'axe de la caméra a changé. Celui de Greenaway est strictement parallèle à la course placide du duc de Milan, et si le réalisateur déclare devant la caméra de Philippe Pilard que le duc c'est l'acteur anglais Gielgud, mais aussi Shakespeare - « des manifestations de la même idée ${ }^{43}$ » - il a l'élégance de s'exclure d'une configuration condensée de postures au sein de laquelle il figure, évidemment. Prospero est bien le personnage délégué du metteur en scène, et d'autant plus que la fonction de maître de cérémonie aux pouvoirs démiurgiques est explicite : de l'ordre d'une conscience tout aussi claire que manifeste sur ce point.

Cette matrice du travelling inaugural ne se laisse jamais complètement oublier, tout au long du film, dès lors qu'il se manifeste plus ou moins occasionnellement "à la marge" des livres inscrits dans le même moule d'une surface parallèle à celle de l'écran, celle surcadrée en son centre. Car cette surface est une zone de surcadrage qui, certes, masque ce qu'elle recouvre, mais dont la couronne rectangulaire périphérique n'annule cependant pas les scènes qui continuent de se dérouler selon l'axe de la profondeur de champ. Cette surface est vue de la salle, mais on extrapole aisément un point de vue de l'envers offert aux personnages qui continuent de déambuler derrière elle. Même s'il 
s'agit d'une pièce rapportée au montage, de nature composite, tout se passe comme si le livre ainsi tendu devenait l'entre-deux de la salle mondaine de cinéma et de l'univers cubique où évoluent les personnages. Le livre incrusté aurait, dans cette hypothèse, la même fonction que celle de la grande toile de Viala qui flotte dans l'embrasure, à la jonction de deux salles du MAMAC de Strasbourg: la "surface-creuset" n'est alors pas antinomique d'un "support-surface" et là est précisément le tour de force de cette singularité de Prospero's Books. Sur cette grande toile surcadrant l'écran de Greenaway, nous assistons à divers événements et processus. La toile devient creuset quand elle est lieu de surgissements organiques mouvementés et colorés, comme dans l'extrait des viandes déjà évoqué et sur lequel revient Eve Ramboz dans le documentaire de Philippe Pilard. Elle devient aussi réceptacle, à l'égal du plan d'eau où plongent aussi quelques personnages de Prospero et la mémoire artistique du spectateur ne manque pas d'établir sur ce point la corrélation entre le film de Greenaway et le vidéo-art de Billl Viola Reflecting Pool ${ }^{44}$. Il semble bien que chez Greenaway, la piscine soit précisément, comme l'encrier où l'écrivain trempe la plume, le lieu de l'affirmation du "devenir-signe" du corps humain, même s'il peut-être voué à disparaître comme chez Viola (relativement et peut-être pas "à tout jamais" s'il s'agit de ne plus apparaitre à l'écran : face au vidéo-art cité, on assiste aussi des réapparitions fantomatiques). Il y a aussi quelque chose de présocratique dans la volonté de Greenaway de convoquer les éléments fondamentaux. Dans le fragment présenté de la forme-balade du travelling, la petite maquette de bateau renversée par un jet d'eau en fin de générique affiche sa facticité, en même temps qu'elle contribue à l'exaltation de l'élément naturel affichée dès la présentation inaugurale du premier livre, dit précisément « livre de l'eau », dans la longue séquence de prégénérique.

Avant que le générique ne s'achève par l'envolée des feuilles de papier qui, après la force de l'eau, exalte celle du vent, on voit Prospero écrire sur une page vierge, et en grandes lettres, "we split, we split, we split ». Couper, diviser, déconstruire pour bâtir autrement, tel est bien ici un principe cinématographique fondamental de l'art de Greenaway affirmé par son personnage délégué. Cependant, nul besoin chez lui du procédé du splitscreen comme solution de montage, procédé qui fit fureur dans la modernité des années 60-70 et qui revient aujourd'huii ${ }^{45}$ : le surcadrage de Prospero est un artifice qui s'inscrit plus naturellement dans le corps même de l'image-mouvement et qui contribue, paradoxalement, à creuser la surface jusqu'à vaincre sa planéité. Dès lors les livres de Prospero sont bien de véritables machines d'hybridation, laboratoires de macération de formes surabondantes, comme dans la toute dernière série de brefs éclats qui enchaine à profusion explosions, effacements, combustions "magiques" de livres dans l'eau de la piscine, événement réitéré (plein cadre) de bris de verre etc.

Dans ces brefs éclats il y a une séduction sensible à laquelle Matthias Müller et Christoph Girardet cèdent en 2006 dans leur œuvre appropriationniste Kristall. Ce film composite est formé de formes brèves analogues empruntées à cent trente huit films, dont de très nombreux grands classiques, comme Müller l'avait déjà fait en 1991 pour Home stories. Kristall organise en séries accumulatives des mouvements, déplacements et gestes semblables, séries présentées comme des «épisodes» transitionnés au noir (fade out + fade in). En accord avec le titre du film, on y trouve de nombreuses fractures, plein cadre, de vitres, verres et autres miroirs. Sous cette clause du verre éclaté en gros plan, il y a une filiation évidente entre Kristall et Prospero's Books, laquelle remonte au film Orphée de Jean Cocteau (1950), auquel Kristall emprunte son dernier plan. 
En 1991, les machines de Greenaway, véritables machineries visuelles, tout autant broyeuses que génératrices, offrent leurs nouveautés créatrices au regard, bien loin d'une esthétique cinématographique pauvre ou banale qui se bornerait à représenter.

\section{BIBLIOGRAPHIE}

- BERGALA (Alain), «Week-end, Le travelling le plus long », in Godard au travail, les années 60, Paris, Cahiers du cinéma, « un réalisateur au travail », 2006.

- BERTHOMÉ (Jean-Pierre), « Hitchcock et Welles », in Dossier Hitchcock de la revue Positif $n^{\circ} 470$ d'avril 2000.

- CAVELL (Stanley), La projection du monde, traduction française de Christian Fournier, Paris,Belin, 1999. (Stanley Cavell, The World Viewed. Reflections on the ontology of film, éd. Harvard University Press, 1971, 1974, 1979).

- DELEUZE (Gilles), ch. 4, « Le corps, la viande et l'esprit, le devenir-animal » in Francis Bacon, logique de la sensation, Paris, Seuil, « l'ordre philosophique », rééd. mai 2002.

- GERSTENKORN (Jacques),La métaphore au cinéma (les figures d'analogie dans les films de fiction,) Paris, éditions Méridiens Klincksieck, 1995.

- FOUCAUlT (Michel), Les Mots et les Choses, Paris, N.R.F. - Gallimard, « Bibliothèque des Sciences Humaines », 1966.

- GREENAWAY (Peter), Quand l'image a le dernier mot, interview par John O’Toole in $n^{\circ} 202$ de la revue artpress,Paris, mai 1995.

- HORS-SERIE « Greenaway », Paris, Le journal Libération, 1987,

- HUYGUE (Pierre), Interviewé par Richard Leydier, Paris, art press nº 322, avril 2006.

- LEUTRAT (Jean-Louis), Le cinéma en perspective : une histoire, éd. Nathan, 1992.

- LEUTRAT (Jean-Louis), « Ouvert pour cause d'inventaire », in La Ville au cinéma, Arras, éd. Artois presses Université, « Cinémas », 2005.

- LEUTRAT (Jean-Louis) et LIANDRAT-GUIGUES (Suzanne), Godard simple comme bonjour, éd.

L'Harmattan, coll. « Esthétiques », Paris, 2004.

- LIANDRAT-GUIGUES (Suzanne), «L'homme qui marche, une allégorie dynamique », Esthétique du mouvement cinématographique, Paris, Klilncksieck, « 50 questions », 2005.

- LIANDRAT-GUIGUES (Suzanne), Cinéma et sculpture, Un aspect de la modernité des années soixante, Paris, L'Harmattan, coll. « L'art en bref », 2002.

- LIANDRAT-GUIGUES (Suzanne), Modernes flâneries du cinéma, De l'incidence éditeur, Lille, 2009.

- LOUGUET (Patrick), « D'un concept, l'autre...un mouvement constant », Murmure Hors-série : Le mouvement des concepts (esthétique - cinéma), Lille, De l'incidence éditeur, 2008. 
- LOUGUET (Patrick), ch. 1 « De la confrontation des esthétiques à une esthétique de la confrontation » in Sensibles Proximités, les arts aux carrefours (cinéma-danse-installation-vidéo-art), Arras, Artois Presse Université, octobre 2009.

- NOGUEZ (Dominique), Ce que le cinéma nous donne à désirer, éditions Yellow Now, Liège, 1996

- NYMAN (Michael), Peter Greenaway, Paris, Dis-Voir, 1987.

- SAVELLI (Julie), « cinéma et anthropologie, l'homme imaginaire en 3 mouvements », Murmure hors-série, Le mouvement des concepts (Esthétique-Cinéma), Lille, De l'incidence éditeur, 2008.

- ZABUNYAN (Dork) « Un détour par la "forme-balade”, in Murmure Hors-série, Le mouvement des concepts, (esthétique - cinéma), Lille, De l'incidence éditeur, 2008.

\section{NOTES}

1. Chez BAC-Video

2. Produit par Ciné Cinéma / C.L.C., (C) Avril 2004.

3. Pierre Huygue, en réponse à l'interviewer, Richard Leydier : "Qu'est-ce que vos films ont que ceux des autres vidéastes n'ont pas? » Paris, artpress n³22, avril 2006, p. 30.

4. Né en 1919 et mort en 2001. Pour Greenaway, avant Prospero's Books, il a déjà cadré et éclairé Le Ventre de l'architecte (1986), Drowning by Numbers (1988), Le Cuisinier, le voleur sa femme et son amant (1989).

5. The Tulse Luper Suitcases, a Personnal History of Uranium.

6. Dans dans le cadre de l'opération «Lille, capitale de la culture", lesdites valises étaient installées dans l'église dont l'abside centrale, cylindrique à chapelles rayonnantes, est surmontée d'une vaste coupole (église Marie-Madeleine, au cœur du vieux Lille, devenue un des hauts lieux lillois des expositions d'Art contemporain avec le Tri Postal et La Gare Saint sauveur : après Lille 2004 capitale européenne de la culture, Lille 3000 XXL en 2009). Les visiteurs étaient invités à arpenter, bottes de caoutchouc aux pieds, cette monumentale installation construite ad hoc au centre de l'église: les valises soutenues par des câbles métalliques, au dessus du plan d'eau circulaire

7. Installée dans une demeure cossue de l'architecture flamande de la fin du XIXe siècle, située au cœur du quartier du parc de la citadelle

8. Suzanne Liandrat-Guigues, Modernes flâneries du cinéma, De l'incidence éditeur, Lille, 2009

(cf. www.wix.com/delincidence/delincidence (taper 2 fois « de l'incidence »).

9. Le journal Libération, en 1987, lui a consacré un hors-série. Cette référence fait partie de la compilation d'articles que l'on trouve sur l'Internet via «Alice-M. Cinéma » sous la requête «Greenaway "

10. Certains vont jusqu'à se demander si Miranda n'est-pas elle-même une invention du duc, au point que dans l'exercice de ses pouvoirs d'exaltation poétique et de transformations magiques, Prospero est affiché nettement par Greenaway comme son personnage délégué, la figure du créateur, son éminent représentant au sein de l'intra-filmique.

11. Michel Foucault, Les Mots et les Choses, Paris, N.R.F. - Gallimard, « Bibliothèque des Sciences Humaines », 1966.

12. Le générique indique bien que le film est réalisé d'après La Tempête de William Shakespeare.

13. Ici, les occurrences canailles le sont du côté des bricolages artisanaux (bricolages à entendre au sens levis-straussien, évidemment).

14. Stanley Cavell, La projection du monde, traduction française de Christian Fournier, Paris, Belin, 1999. L'esthéticien du cinéma s'appuie sur le Salon de Baudelaire rédigé en 1859, p. 74 (Stanley Cavell, The World Viewed. Reflections on the ontology of film, éd. Harvard University 
Press, 1971,1974,1979). Voir aussi Jean-Louis Leutrat, Le cinéma en perspective : une histoire, éd. Nathan, 1992.

15. Sur cette question des intercesseurs, voir mon article « D'un concept, l'autre...un mouvement constant » in Murmure Hors-série : Le mouvement des concepts (esthétique - cinéma), Lille, 2008, p. 107.

16. La connivence moderne entre l'art de Greenaway et celui de Shakespeare fait bien du dramaturge le contemporain du cinéaste et, par sa médiation, notre contemporain. C'est que cette connivence, ou grande affinité active et réactives, ses « actualités intempestives ", dit à la manière de Nietzsche pour caractériser tout ce qui insiste à travers l'Histoire - ou malgré elle - et pour désigner ce que les hommes ne peuvent jamais oublier, même s'ils le tentent parfois avec mauvaise-foi. Ainsi en est-il des combinaisons entre violences crues et raffinées dans les films de Greenaway.

17. Le très grand écran est, avec la projection lumineuse, une des conditions essentielles du cinéma. Voir Dominique Noguez, Ce que le cinéma nous donne à désirer, éditions Yellow Now, Liège, 1996.

18. Épisode fameux rapporté par Micky Kwella en 1994 dans le documentaire Théma de la chaîne télévisuelle arte intitulé Tempête d'images.

19. Joseph Leo Mankiewicz, L'aventure de Madame Muir (The Ghost and Mrs Muir - 1947).

20. Jacques Gerstenkorn, La métaphore au cinéma (les figures d'analogie dans les films de fiction,) Paris, éditions Méridiens Klincksieck, 1995.

21. Cette circulation ou (autre solution) cet effeuillage, bien que cliché de cinéma, est toujours source d'une émotion sensible.

22. En cela la profonde connivence de l'esthétique cinématographique de Peter Greenaway avec l'esthétique musicale de Michael Nyman.

23. Peter Greenaway, Quand l'image a le dernier mot, interview par John O'Toole in $n^{\circ} 202$ de la revue artpress, Paris, mai 1995, p. 22-30. Le propos cité se trouve p. 24.

24. Ne serait-ce que celles de décors grandioses, aux détails luxueux, tels que les exaltent les frères Paolo et Vittorio Taviani dans leur film Good Morning Babylone (Good Morning Babilonia 1987).

25. Ibidem.

26. Rebecca (1940) ; Citizen Kane (1941). Voir Jean-Pierre Berthomé in revue Positif $\mathrm{n}^{\circ} 470$ d'avril 2000, p. 76 à 81. Son article est intitulé significativement "Hitchcock et Welles » au sein du Dossier Hitchcock figurant dans ce numéro. L'article de Berthomé qui s'intéresse à de troublantes proximités esthétiques de deux grands maîtres qui affectaient de s'ignorer est sous-titré avec un mot d'Orson Welles : «Je l'aimais beaucoup, il n'aimait pas mes films non plus ».

27. Jean-Claude Biette cité par Julie Savelli dans son article « cinéma et anthropologie, l'homme imaginaire en 3 mouvements", in Murmure hors-série, Le mouvement des concepts (esthétique - cinéma), Lille, De l'incidence éditeur, février 2008, p. 134.

28. Ibidem.

29. Question affirmée constamment par Greenaway dans ses œuvres, en accord avec le propos de 1995 rapporté.

30. Suzanne Liandrat-Guigues, Cinéma et sculpture, Un aspect de la modernité des années soixante, Paris, L'Harmattan, coll. « L'art en bref », 2002.

31. Dans le livre 18 de Prospero intitulé The autobiographies of Sémiramis and Paphinae.

32. Voir le DVD consacré à Méliès, Méliès, le cinémagicien, présenté par Madeleine MalthêteMéliès, éd. arte vidéo, EDV 236, 2001.

33. Voir plus particulièrement Gilles Deleuze, chapitre 4, «Le corps, la viande et l'esprit, le devenir-animal » in Francis Bacon, logique de la sensation, Paris, Seuil, «l'ordre philosophique », rééd. mai 2002, p. 27.

34. Dans sa communication donnée en décembre 2008 à l'Université de Montpellier 3. 
35. Michael Nyman, Peter Greenaway, Paris, Dis-Voir, 1987, p. 40. (Le dernier film à l'époque de cette parution était Le Ventre de l'architecte (The Belly of an Architect - 1987).

36. La forme-bal(l)ade, à la suite de Gilles Deleuze, fait partie de concepts esthétiques centraux de Suzanne Liandrat-Guiges. Voir «L'homme qui marche, une allégorie dynamique », Esthétique du mouvement cinématographique, Paris, Klilncksieck , «50 questions», 2005, p. 111 et, plus particulièrement, "Que nous apprend la forme «bal(l)ade?», p. 133. Voir aussi Dork Zabunyan "Un détour par la "forme-balade", in Murmure Hors-série, Le mouvement des concepts, (esthétique - cinéma), Lille, De l'incidence éditeur, 2008, p.23-33.

37. Comme Suzanne Liandrat-Guigues le dit de la flânerie dans le chapitre «créatures du passage ", Modernes flâneries, op.cit., p. 37.

38. Voir les œuvres de Félicien Rops au musée belge de Namur consacré à cet artiste.

39. Sur l'ekphrasis, voir Suzanne Liandrat-Guigues et Jean-Louis Leutrat, Godard simple comme bonjour, éd. L'Harmattan, coll. 'Esthétiques', Paris, 2004, p. 126. Voir aussi Patrick Louguet, ch. 1 " de la confrontation des esthétiques à une esthétique de la confrontation » in Sensibles Proximités, les arts aux carrefours (cinéma-danse-installation-vidéo-art), Arras, Artois Presse Université, octobre 2009, p. 31.

40. Sur cette question de l'hybridation des Nevers et d'Hiroshima, comme «deux peignes entremêlés ", voir Jean-Louis Leutrat, "Ouvert pour cause d'inventaire ", in La Ville au cinéma, Arras, éd. Artois presses Université, « Cinémas », 2005, p. 241.

41. Alain Bergala, "Week-end ", "Le travelling le plus long ", in Godard au travail, les années 60, Paris, Cahiers du cinéma, « un réalisateur au travail », 2006, p. 372.

42. Dit-il dans son interview par Philippe Pilard et Jean-Jacques Bernard dans leur documentaire de 2004.

43.

44. Reflecting Pool est un vidéo-art réalisé réalisée en 1983 par Bill Viola. Un extrait de cette œuvre figure à l'intérieur du documentaire artistique écrit et réalisé par Aude de Laforcade, intitulé tout simplement L'Art vidéo et diffusé sur la chaîne 5 de la télévision française en Automne 2001.

45. Citons, dans l'arbitraire d'une énumération économique, le film de John Frankenheimer de 1966, Grand Prix; celui de Norman Jewison, L'affaire Thomas Crown (The Thomas Crown Affair -1968) ; pour ce qu'il en est des années 60-70 puis, pour les années 90 : Denise au téléphone (Denise Calls up ! - 1994) de Hal Salwen; Cours Lola cours (Lola rennt- 1998) de Tom Tykwer et Time Code de Mike Figgis (2000).

\section{AUTEUR}

\section{PATRICK LOUGUET}

Université d'Artois, Praxis et esthétique des arts, E.A 4028, Fr - 62000 Arras 